\title{
TWO-DIMENSIONAL DEFORMATION IN A THERMOELASTIC SOLID WITH MICROTEMPERATURES SUBJECTED TO AN INTERNAL HEAT SOURCE
}

\author{
P. AILAWALIA* \\ Department of Applied Sciences, Maharishi Markandeshwar University \\ Sadopur, Ambala City, Haryana, INDIA \\ E-mail: praveen_2117@rediffmail.com \\ S. BUDHIRAJA \\ Department of Applied Sciences, Chandigarh Group of Colleges \\ Landran, Mohali, Punjab, INDIA \\ Punjab Technical University \\ Jalandhar, Punjab, INDIA \\ J. SINGH \\ Department of Applied Sciences, Chandigarh Group of Colleges \\ Landran, Mohali, Punjab, INDIA
}

\begin{abstract}
The purpose of this paper is to study the two dimensional deformation in a generalized thermoelastic medium with microtemperatures having an internal heat source subjected to a mechanical force. The force is acting along the interface of generalized thermoelastic half space and generalized thermoelastic half space with microtemperatures having an internal heat source. The normal mode analysis has been applied to obtain the exact expressions for the considered variables. The effect of internal heat source and microtemperatures on the above components has been depicted graphically.
\end{abstract}

Key words: thermoelasticity, internal heat source, normal-mode, microtemperatures.

\section{Introduction}

In recent years the theory of thermoelasticity for bodies with microstructures has been intensively studied. Grot [1] was the first who developed the concept of microtemperatures and established a theory of thermodynamics for elastic materials with microstructure. The particles of an elastic material with an innerstructure possess microtemperatures. He modified the second law of thermodynamics to include microtemperatures. Iesan and Quintanilla [2] introduced a linear theory for elastic materials with an inner structure whose particles, in addition to the classical displacement and temperature fields, possess microtemperature, and established the continuous dependence of solutions on the initial data and body loads. They modified the Clausius-Duhem inequality to include microtemperatures and also the first order moment of the energy equations are added to the usual balance laws of a continuum with microstructures. Riha [3] applied a theory of heat-conducting micromorphic continua to an analysis of heat conduction in materials with inner structures so that it is proved that the experimental data for the silicone rubber containing aluminum particles and for human blood conform closely to the predicted theoretical model of thermoelasticity with microtemperatures. Riha $[4,5]$ studied the theory of heat-conducting micropolar fluids

\footnotetext{
${ }^{*}$ To whom correspondence should be addressed
} 
with microtemperatures and solved the problem of the Poiseuille flow between two parallel plates, while Iesan $[6,7]$ developed a linear theory for elastic materials with an inner structure whose particles, in addition to the classical displacement and temperature, possess microtemperatures and can stretch as well as contract independently of their translations. The theory of thermoelastic bodies with microstructures and microtemperatures was introduced by Iesan [8]. In this theory, the microelements of the material possess microtemperatures and can undergo microrotation, microstretch and translation. He also showed that there exists coupling of microrotation vectors fields with microtemperatures even for isotropic bodies. Recently, Iesan and Quintanilla [9] presented a linear theory of thermoelastic bodies with a microstructure and microtemperatures which permits the transmission of heat as thermal waves at finite speed. They established the existence and uniqueness results in the context of the dynamic theory. Svanadze $[10,11]$ studied the fundamental solutions to the equations of the equilibrium and investigated the steady oscillations of the theory of thermoelasticity with microtemperatures. Scalia and Svanadze [12, 13] considered the linear theory of thermoelasticity with microtemperatures and investigated the basic boundary value problems of steady vibrations by using the potential method. Scalia et al. [14] studied some fundamental solutions and proved the existence and uniqueness theorems for equilibrium solutions and steady state vibrations by means of the potential method. Iesan [15] studied the behavior of shock waves and higher-order discontinuities which propagate in a thermoelastic body with an inner structure and microtemperatures, while Iesan and Scalia [16] investigated the plane strain of homogeneous and isotropic elastic solids and the problem of thermal stresses in an elastic space with a cylindrical hole. Yang and Huang [17] studied the propagation of singularities of solutions to the Cauchy problem of a semilinear thermoelastic system with microtemperatures in one space variable. Some basic theorems are also developed by Aouadi [18] and Svanadze and Tracina [19] in which they considered the linear theory of microstretch thermoelasticity for isotropic solids with microtemperatures. Chirita et al. [20] studied the linear theory of thermoelastic materials with an inner structure whose particles, in addition to the classical displacement and temperature fields, possess microtemperatures. Steeb et al. [21] investigated the propagation of time harmonic waves in an infinite thermoelastic medium with microtemperatures. Kumar and Kaur [22] studied the reflection and refraction of plane waves at the interface of an elastic solid and microstretch thermoelastic solid with microtemperatures. Ailawalia et al. [23] studied the two dimensional deformation in a microstretch thermoelastic half space with microtemperatures and an internal heat source. Othman et al. [24] studied the effect of the gravity field on an initially stressed micropolar thermoelastic medium with microtemperatures.

In the present problem, the authors have discussed the deformation in a generalized thermoelastic medium with microtemperature having an internal heat source subjected to a mechanical force. Using the normal mode analysis method, we get the analytical expressions for the displacement component, force stress and temperature distribution. Variations of the considered variables through the vertical distance are illustrated graphically to show the effect of microtemperatures and the internal heat source on the displacement components, force stresses and temperature distribution.

\section{Formulation of the problem}

We consider a mechanical force of magnitude $F$ acting along the interface of a generalized thermoelastic medium with microtemperatures and an internal heat source (medium I) occupying the region $0 \leq z \leq \infty$ and a generalized thermoelastic medium (medium II) occupying the region $-\infty<z<0$. The plane $z=0$ represents the interface of medium I and medium II.

A rectangular coordinate system $(x, y, z)$ with the $z$-axis pointing vertically downward is considered. Since we are considering a plane strain problem in the $x z$-plane, hence all quantities considered are functions of the time variable $t$ and of the coordinates $x$ and $z$. 


\section{Basic equations and their solutions}

Following Iesan and Quintanilla [2], the constitutive relations for a homogeneous and isotropic thermoelastic medium with microtemperatures and an internal heat source are

$$
\begin{aligned}
& t_{i j}^{\diamond}=\lambda^{\diamond} \delta_{i j} e_{r r}^{\diamond}+2 \mu^{\diamond} e_{i j}^{\diamond}-\beta^{\diamond} T^{\diamond} \delta_{i j}, \\
& q_{i}=K^{* \diamond} T_{, i}^{\diamond}+k_{1} w_{i}, \\
& q_{i j}=-k_{4} w_{r, r} \delta_{i j}-k_{5} w_{i, j}-k_{6} w_{j, i}, \\
& Q_{i}=\left(k_{1}-k_{2}\right) w_{i}+\left(K^{* \diamond}-k_{3}\right) T_{, i}^{\diamond}, \\
& \rho^{\diamond} \eta^{* \diamond}=\beta^{\diamond} e_{r r}{ }^{\diamond}+a^{\diamond} T^{\diamond}, \\
& \rho^{\diamond} \varepsilon_{i}=-b w_{i},
\end{aligned}
$$

where

$$
e_{i j}^{\diamond}=\frac{1}{2}\left(u_{i, j} \diamond+u_{j, i} \diamond\right)
$$

and $\lambda^{\diamond}, \mu^{\diamond}, \beta^{\diamond}, a^{\diamond}, b, K^{*}, k_{i}(j=1,2, \ldots .6)$ are constant constitutive coefficents, $t_{i j}{ }^{\ominus}$ are the components of stress tensor, $\rho^{\diamond}$ is the reference mass density of the medium, $\eta^{*}$ is the entropy per unit mass, $\varepsilon_{i}$ are the components of the first moment of the energy vector, $q_{i j}$ are the components of the first heat flux moment vector. $q_{i}$ are the components of the heat flux vector, $u_{i}{ }^{\circ}$ are the components of the displacement vector $\boldsymbol{u}^{\diamond}$, $w_{i}$ are the components of the microtemperatures vector $\boldsymbol{w}, T^{\diamond}=\Theta-T_{0}$, where $\Theta$ is the temperature at time $t, T_{0}$ is the temperature of the medium in its natural state and assumed to be such that $\left|T^{\diamond} / T_{0}\right| \leq 1$. A comma in the subscript denotes the spatial derivative and $\delta_{i j}$ is the Kronecker delta.

Following Iesan and Quintanilla [2], the consitutive Eqs (3.1)-(3.3), combined with the reduced Clausius-Duhem inequality in the context of the linear theory of thermoelasticity with microtemperatures imply the following inequalities

$$
\begin{aligned}
& 3 k_{4}+k_{5}+k_{6} \geq 0, \quad k_{6}+k_{5} \geq 0, \\
& k_{6}-k_{5} \geq 0, \quad K^{* \diamond} \geq 0, \\
& \left(k_{1}+T_{0} k_{3}\right)^{2}-4 T_{0} K^{* \diamond} k_{2} \leq 0 .
\end{aligned}
$$

Following Iesan and Quintanilla [2], the fundamental system of field eqautions of the linear theory of thermoelasticity with microtemperatures and an internal heat source in the absence of body force are 
(i) Stress equation of motion

$$
t_{j i, j}{ }^{\diamond}=\rho^{\diamond} \ddot{u}_{i}
$$

(ii) Equation of balance of energy

$$
\rho^{\diamond} T_{0} \eta^{\diamond}=q_{i, i}+Q
$$

where $Q$ is the stable internal heat source.

(iii) Equation of balance of the first moment of energy

$$
\rho^{\diamond} \dot{\varepsilon}_{i}=q_{j i, j}+q_{i}-Q_{i}
$$

where superposed dot represents the temporal derivative and other symbols are as described previously.

Using Eqs (3.1)-(3.6) in Eqs (3.10)-(3.12), the following system of partial differential equations is obtained

$$
\begin{aligned}
& \mu^{\diamond} u_{i, j j}{ }^{\diamond}+\left(\lambda^{\diamond}+\mu^{\diamond}\right) u_{j, i j}{ }^{\diamond}-\beta^{\diamond} T_{, i}^{\diamond}=\rho^{\diamond} \ddot{u}_{i}^{\diamond}, \\
& K^{* \diamond} T_{, i i}-\beta^{\diamond} T_{0} \dot{u}_{i, i}^{\diamond}+k_{1} w_{i, i}=a^{\diamond} T_{0} \dot{T}^{\diamond}+Q, \\
& k_{6} w_{i, j j}+\left(k_{4}+k_{5}\right) w_{j, j i}-k_{3} T_{, i}^{\diamond}-k_{2} w_{i}=b \dot{w}_{i} .
\end{aligned}
$$

For the generalized thermoelastic half-space with microtemperatures having an internal heat source (Medium I) $\diamond$ is replaced by I. For the generalized thermoelastic half-space (Medium II) $\diamond$ is replaced $y$ II with $k_{1}=k_{2}=k_{3}=k_{4}=k_{5}=k_{6}=Q=0$.

For the two dimensional problem, all quantities considered are functions of the time variable $t$ and of the coordinates $x$ and $z$. The displacement vector $\boldsymbol{u}^{I}$ and microtemperature vector $\boldsymbol{w}$ are thus taken as

$$
\boldsymbol{u}^{I}=\left(u_{1}^{I}(x, z, t), 0, u_{3}^{I}(x, z, t)\right), \quad \boldsymbol{w}=\left(w_{1}(x, z, t), 0, w_{3}(x, z, t)\right)
$$

For convenience, the following non-dimensional variables are used

$$
\begin{aligned}
& \left(x^{\prime}, z^{\prime}\right)=\frac{1}{L}(x, z), \quad\left(u_{1}^{I^{\prime}}, u_{3}^{I^{\prime}}\right)=\frac{1}{L}\left(u_{1}^{I}, u_{3}{ }^{I}\right), \quad t_{i j}^{I^{\prime}}=\frac{1}{\beta^{\diamond} T_{0}} t_{i j}{ }^{I}, \quad q_{i j}{ }^{\prime}=\frac{1}{L c_{1} \beta^{I} T_{0}} q_{i j}, \\
& t^{\prime}=\frac{c_{1}}{\mathrm{~L}} t, \quad T^{I^{\prime}}=\frac{T^{I}}{T_{0}}, \quad w_{i}^{\prime}=L w_{i}, \quad Q^{\prime}=\frac{Q}{Q_{0}}, \quad c_{1}^{2}=\frac{\lambda^{I}+2 \mu^{I}}{\rho^{I}}
\end{aligned}
$$

where $L$ is a parameter having dimension of length.

Using relations in terms of scalar potential functions $\varphi^{I}(x, y, t), \psi^{I}(x, y, t), \phi_{1}(x, y, t)$ and $\psi_{1}(x, y, t)$ defined by 


$$
u_{1}^{I}=\frac{\partial \varphi^{I}}{\partial x}-\frac{\partial \psi^{I}}{\partial z}, \quad u_{3}^{I}=\frac{\partial \varphi^{I}}{\partial z}+\frac{\partial \psi^{I}}{\partial x}, \quad w_{1}=\frac{\partial \phi_{1}}{\partial x}-\frac{\partial \psi_{1}}{\partial z}, \quad w_{3}=\frac{\partial \phi_{1}}{\partial z}+\frac{\partial \psi_{1}}{\partial x}
$$

and dimensionless variables given by Eq.(3.17) in Eqs (3.13)-(3.15), we obtain the following dimensionless equations (after dropping superscripts)

$$
\begin{aligned}
& \left(\frac{\partial^{2}}{\partial x^{2}}+\frac{\partial^{2}}{\partial z^{2}}-\frac{\partial^{2}}{\partial t^{2}}\right) \varphi^{I}-\xi_{3} T^{I}=0 \\
& \left(1+\xi_{7}\right)\left(\frac{\partial^{2}}{\partial x^{2}}+\frac{\partial^{2}}{\partial z^{2}}\right) \phi_{1}-\xi_{8} \frac{\partial \phi_{1}}{\partial t}-\xi_{9} \phi_{1}-\xi_{10} T^{I}=0 \\
& \left(\frac{\partial^{2}}{\partial x^{2}}+\frac{\partial^{2}}{\partial z^{2}}\right) T^{I}-\xi_{4} \frac{\partial T^{I}}{\partial x}-\xi_{5}\left(\frac{\partial^{2}}{\partial x^{2}}+\frac{\partial^{2}}{\partial z^{2}}\right) \frac{\partial \varphi^{I}}{\partial t}+\xi_{6} \nabla^{2} \frac{\partial \phi_{1}}{\partial t}=B^{*} Q \\
& \left(\xi_{1} \frac{\partial^{2}}{\partial x^{2}}+\xi_{1} \frac{\partial^{2}}{\partial z^{2}}-\frac{\partial^{2}}{\partial t^{2}}\right) \psi^{I}=0 \\
& \left(\frac{\partial^{2}}{\partial x^{2}}+\frac{\partial^{2}}{\partial z^{2}}-\xi_{8} \frac{\partial}{\partial t}-\xi_{9}\right) \psi_{1}=0
\end{aligned}
$$

where

$$
\begin{aligned}
& \xi_{1}=\left(\frac{\mu^{I}}{\lambda^{I}+2 \mu^{I}}\right), \quad \xi_{2}=\left(\frac{\lambda^{I}+\mu^{I}}{\lambda^{I}+2 \mu^{I}}\right), \quad \xi_{3}=\frac{\beta^{I} T_{0}}{\lambda^{I}+2 \mu^{I}}, \quad \xi_{4}=\frac{a^{I} T_{0} c_{1} L}{K^{* I}}, \\
& \xi_{5}=\frac{\beta^{I} c_{1} L}{K^{* I}}, \quad \xi_{6}=\frac{k_{1}}{K^{* I} T_{0}}, \quad \xi_{7}=\frac{k_{4}+k_{5}}{k_{6}}, \quad \xi_{8}=\frac{b_{1} c_{1} L}{k_{6}}, \quad \xi_{9}=\frac{k_{2} L^{2}}{k_{6}}, \\
& \xi_{10}=\frac{k_{3} T_{0} L^{2}}{k_{6}}, \quad \xi_{11}=\left(1+a_{7}\right), \quad \xi_{12}=\xi_{11} a^{2}+\xi_{8} \omega+\xi_{9}, \quad \xi_{13}=1 a \xi_{4}+a^{2}, \\
& \xi_{14}=a^{2}+\omega^{2}, \quad \xi_{15}=\frac{\lambda^{I}}{\beta^{I} T_{0}}, \quad \xi_{16}=\frac{\lambda^{I}+2 \mu^{I}}{\beta^{I} T_{0}}, \quad \xi_{17}=\frac{\mu^{I}}{\beta^{I} T_{0}}, \quad \xi_{18}=\frac{k_{4}+k_{5}+k_{6}}{c_{1} \beta^{I} L^{3} T_{0}}, \\
& \xi_{19}=\frac{-k_{4}}{c_{1} L^{3} \beta^{I} T_{0}}, \quad \xi_{20}=\frac{-k_{5}}{c_{1} L^{3} \beta^{I} T_{0}}, \quad \xi_{21}=\frac{-k_{6}}{c_{1} L^{3} \beta^{I} T_{0}}, \quad B^{*}=\frac{L^{2}}{K^{* I} T_{0}} .
\end{aligned}
$$

\section{Normal mode analysis}

The solution of the considered physical variable can be decomposed in terms of normal modes and can be considered in the following form 


$$
\begin{aligned}
& {\left[\varphi^{I}, \psi^{I}, \phi_{1}, \psi_{1}, t_{i j}{ }^{I}, T^{I}, q_{i j}\right](x, z, t)=\left[\bar{\varphi}^{I}, \bar{\psi}^{I}, \bar{\phi}_{1}, \bar{\psi}_{1}, \bar{t}_{i j}{ }^{I}, \bar{T}^{I}, \bar{q}_{i j}\right](z) e^{(\omega t+1 a x)},} \\
& Q=Q_{0} e^{(\omega t+1 a x)}
\end{aligned}
$$

where $\left[\bar{\varphi}^{I}, \bar{\psi}^{I}, \phi_{1}, \psi_{1}, \bar{T}^{I}, \bar{t}_{i j}^{I}, \bar{q}_{i j}\right]$ are the magnitude of the functions, $\omega$ is the complex time constant and $a$ is the wave number in the $x$-direction and $Q_{0}$ is the magnitude of the stable internal heat source.

Using normal mode in Eqs (3.19)-(3.23) we get

$$
\begin{aligned}
& {\left[\nabla^{2}-a^{2}-\omega^{2}\right] \bar{\varphi}^{I}-\xi_{3} \bar{T}^{I}=0,} \\
& \left(\nabla^{2}-a^{2}\right) \bar{T}^{I}-\xi_{4} 1 a \bar{T}^{I}-\xi_{5} \omega\left(\nabla^{2}-a^{2}\right) \bar{\varphi}^{I}+\xi_{6} \omega\left(\nabla^{2}-a^{2}\right) \bar{\phi}_{1}=B^{*} Q_{0}, \\
& \xi_{11}\left(\nabla^{2}-a^{2}\right) \bar{\phi}_{1}-\xi_{8} \omega \bar{\phi}_{1}-\xi_{9} \bar{\phi}_{1}-\xi_{10} \bar{T}^{I}=0, \\
& {\left[\xi_{1}\left(\nabla^{2}-a^{2}\right)-\omega^{2}\right] \bar{\psi}^{I}=0} \\
& {\left[\nabla^{2}-a^{2}-\xi_{8} \omega-\xi_{9}\right] \bar{\psi}_{1}=0}
\end{aligned}
$$

where

$$
\nabla=\frac{d}{d z}
$$

Eliminating $\bar{\varphi}^{I}$ and $\bar{\phi}_{1}$ from Eqs (4.3)-(4.5) we get the following sixth-order differential equation as

$$
\left[\nabla^{6}-P \nabla^{4}+R \nabla^{2}-S\right]\left(\bar{T}^{I}\right)=l_{51} .
$$

where

$$
\begin{aligned}
& P=\left(\xi_{3} \xi_{5} \omega+\xi_{13}+\xi_{14}\right)+\frac{\xi_{12}}{\xi_{11}}-\frac{\xi_{6} \xi_{10} \omega}{\xi_{11}} \\
& R=\xi_{13} \xi_{14}+\xi_{3} \xi_{5} \omega\left(a^{2}+\frac{\xi_{12}}{\xi_{11}}\right)-\frac{\xi_{6} \xi_{10} \omega\left(a^{2}+\xi_{14}\right)}{\xi_{11}}+\frac{\xi_{12}\left(\xi_{13}+\xi_{14}\right)}{\xi_{11}} \\
& S=\frac{\omega a^{2}\left(\xi_{6} \xi_{10} \xi_{14}-\xi_{3} \xi_{5} \xi_{12}\right)}{\xi_{11}}+\frac{\xi_{12} \xi_{13} \xi_{14}}{\varsigma_{11}} \\
& l_{51}=\frac{B^{*} \xi_{12} \xi_{14} Q_{0}}{\xi_{11}}
\end{aligned}
$$


The solution of Eq.(4.8) has the form

$$
\bar{T}^{I}=\sum_{j=1}^{3} N_{j}(a, \omega) e^{-h_{j} z}+l_{51}
$$

where $h_{j}^{2}(j=1,2,3)$ are the roots of the characteristic Eq.(4.8).

Since $\bar{T}^{I}, \bar{\varphi}^{I}$ and $\bar{\phi}_{1}$ are coupled parameters as shown in Eqs (4.3)-(4.5), therefore we may suppose

$$
\begin{gathered}
\bar{\varphi}^{I}=\sum_{j=1}^{3} \alpha_{5 j} N_{j}(a, \omega) e^{-h_{j} z}+l_{52}, \\
\bar{\phi}_{1}=\sum_{j=1}^{3} \beta_{5 j} N_{j}^{\prime \prime}(a, \omega) e^{-h_{j} z}+l_{53}
\end{gathered}
$$

where $N_{j}(a, \omega), N_{j}^{\prime}(a, \omega)$ and $N_{j}^{\prime \prime}(a, \omega)$ are specific functions depending upon $a, \omega$ and $\alpha_{5 j}$ and $\beta_{5 j}(j=1,2,3)$ are the coupling constants. The values of coupling constants are obtained from Eqs (4.3)(4.5) and are given as

$$
\begin{aligned}
& \alpha_{5 j}=\frac{\xi_{3}}{\left(h_{j}^{2}-a^{2}-\omega^{2}\right)}, \\
& \beta_{5 j}=\frac{\xi_{10}}{\left(\zeta_{11} h_{j}^{2}-\zeta_{12}\right)}, \\
& l_{52}=-B^{*}\left(\xi_{8} \omega+\xi_{9}+\xi_{11} a^{2}\right), \\
& l_{53}=\frac{B^{*} Q_{0} \xi_{10}\left(a^{2}+\omega^{2}\right)}{\xi_{3}} .
\end{aligned}
$$

The solutions of Eqs (4.6) and (4.7) are given by

$$
\begin{aligned}
& \bar{\psi}^{I}=N_{4}(a, \omega) e^{-h_{4} z} \\
& \bar{\psi}_{1}=N_{5}(a, \omega) e^{-h_{5} z}
\end{aligned}
$$

where $N_{4}(a, \omega)$ and $N_{5}(a, \omega)$ are specific functions depending upon $a, \omega$ and $h_{4}$ and $h_{5}$ are the roots of the characteristic Eqs (4.6) and (4.7) respectively given by

$$
h_{4}^{2}=\frac{\xi_{1} a^{2}+\omega^{2}}{\xi_{1}}, \quad h_{5}^{2}=\xi_{9}+\xi_{8} \omega+a^{2} .
$$


Thus the solutions of Eqs (3.13)-(3.15) are

$$
\begin{aligned}
& u_{1}^{I}=\left(\sum_{j=1}^{3} 1 a \alpha_{5 j} N_{j}(a, \omega) e^{-h_{j} z}+h_{4} N_{4}(a, \omega) e^{-h_{4} z}\right) e^{(\omega t+1 a x)}+1 a l_{52}, \\
& u_{3}^{I}=\left(-\sum_{j=1}^{3} h_{j} \alpha_{5 j} N_{j}(a, \omega) e^{-h_{j} z}+1 a N_{4}(a, \omega) e^{-h_{4} z}\right) e^{(\omega t+1 a x)}, \\
& T^{I}=\left(\sum_{j=1}^{3} N_{j}(a, \omega) e^{-h_{j} z}\right) e^{(\omega t+1 a x)}+l_{51}, \\
& t_{31}^{I}=\left(\sum_{j=1}^{3} s_{5 j} N_{j}(a, \omega) e^{-h_{j} z}-s_{54} N_{4}(a, \omega) e^{-h_{4} z}\right) e^{(\omega t+1 a x)}, \\
& t_{33}^{I}=\left(\sum_{j=1}^{3} r_{5 j} N_{j}(a, \omega) e^{-h_{j} z}+r_{54} N_{4}(a, \omega) e^{-h_{4} z}\right) e^{(\omega t+1 a x)}-F_{51}, \\
& q_{33}=\left(\sum_{j=1}^{3} \gamma_{5 j} N_{j}(a, \omega) e^{-h_{j} z}+\gamma_{55} N_{5}(a, \omega) e^{-h_{4} z}\right) e^{(\omega t+1 a x)}-F_{52} \\
& \left.q_{j=1}^{3} \eta_{5 j} N_{j}(a, \omega) e^{-h_{j} z}+\eta_{55} N_{5}(a, \omega) e^{-h_{5} z}\right) e^{(\omega t+1 a x)},
\end{aligned}
$$

where

$$
\begin{aligned}
& r_{5 j}=\alpha_{5 j}\left(\xi_{16} h_{j}^{2}-\xi_{15} a^{2}\right)-1, \quad s_{5 j}=-21 a \alpha_{5 j} \xi_{17} h_{j}, \quad \gamma_{5 j}=\beta_{5 j}\left(\xi_{18} h_{j}^{2}-\xi_{19} a^{2}\right), \\
& \eta_{5 j}=-1 a h_{j} \beta_{j}\left(\xi_{20}+\xi_{21}\right), \quad r_{54}=1 a h_{4}\left(\xi_{15}-\xi_{16}\right), \quad s_{54}=\xi_{17}\left(a^{2}+h_{4}^{2}\right), \\
& \gamma_{55}=1 a h_{5}\left(\xi_{19}+\xi_{18}\right), \quad \gamma_{55}=1 a h_{5}\left(\xi_{19}-\xi_{18}\right), \\
& F_{51}=-\left(l_{51}+\xi_{15} a^{2} l_{52}\right), \quad F_{52}=a_{19} a^{2} l_{53} .
\end{aligned}
$$

Neglecting the effect of microtemperatures i.e., $k_{1}=k_{2}=k_{3}=k_{4}=k_{5}=k_{6}=0$ and letting $Q \rightarrow 0$ in Eqs (3.14)-(3.15), we obtain the field equations and their constitutive relations in a generalized thermoelastic medium. Adopting the same methodology, the expression for displacement components $\left(u_{1}^{I I}\right.$, $\left.u_{3}^{I I}\right)$, force stresses $\left(t_{33}^{I I}, t_{31}^{I I}\right)$ and temperature distribution $T^{I I}$ for the generalized thermoelastic medium (medium II) are obtained as 


$$
\begin{aligned}
& \bar{u}_{1}^{I I}=\left(\sum_{n=1}^{2} 1 a a_{5 n} R_{n}(a, \omega) e^{\lambda_{n^{z}}}-\lambda_{3} R_{3} e^{\lambda_{3} z}\right) \\
& \bar{u}_{3}^{I I}=\left(\sum_{n=1}^{2} \lambda_{n} a_{5 n} R_{n}(a, \omega) e^{\lambda_{n} z}+1 a R_{3} e^{\lambda_{3} z}\right) \\
& {\overline{t_{33}}}^{I I}=\left(\sum_{n=1}^{2} g_{5 n} R_{n}(a, \omega) e^{\lambda_{n^{z}}}+L_{51} R_{3} e^{\lambda_{3} z}\right) \\
& \bar{t}_{31}^{I I}=\left(\sum_{n=1}^{2} q_{5 n} R_{n}(a, \omega) e^{\lambda_{n^{z}}}+L_{52} R_{3} e^{\lambda_{3} z}\right) \\
& \bar{T}^{I I}=\left(\sum_{n=1}^{2} R_{n}(a, \omega) e^{\lambda_{n^{z}}}\right)
\end{aligned}
$$

where $\left[\bar{u}_{1}^{I I}, \bar{u}_{3}{ }^{I I}, \bar{t}_{31}{ }^{I I}, \bar{t}_{33}{ }^{I I}, \bar{T}^{I I}\right](z) e^{(\omega t+\mathrm{lax})}=\left[u_{1}{ }^{I I}, u_{3}{ }^{I I}, t_{31}{ }^{I I}, t_{33}{ }^{I I}, T^{I I}\right](x, z, t)$ and $R_{n}(a, \omega),(n=1,2)$ are the specific functions depending upon $a, \omega . \lambda_{n}^{2}(n=1,2)$ are the roots of the characteristic equation

$$
\left[\nabla^{4}-U \nabla^{2}+V\right]\left(\bar{T}^{I I}\right)=0
$$

with

$U=\left(2 a^{2}+b_{56} 1 a+\omega\left(\omega+b_{52} b_{57}\right)\right), \quad V=\left(a^{2}\left(a^{2}+b_{56} 1 a+b_{52} b_{57} \omega\right)+\omega^{2}\left(a^{2}+b_{56} 1 a\right)\right)$ and $\lambda_{3}^{2}$ are the roots of the characteristic equation

$$
\left[b_{51}\left(\nabla^{2}-a^{2}\right)-\omega^{2}\right] \bar{\psi}^{I I}=0
$$

where

$$
\begin{aligned}
& b_{51}=\left(\frac{\mu^{I I}}{\lambda^{I I}+2 \mu^{I I}}\right), \quad b_{52}=\frac{\beta^{I I} T_{0}}{\lambda^{I I}+2 \mu^{I I}}, \quad b_{53}=\frac{\lambda^{I I}+2 \mu^{I I}}{\beta^{I I} T_{0}}, \quad b_{54}=\frac{\lambda^{I I}}{\beta^{I I} T_{0}}, \\
& b_{55}=\frac{\mu^{I I}}{\beta^{I I} T_{0}}, \quad b_{56}=\frac{a^{I I} T_{0} c_{1} L}{K^{* I}}, \quad b_{57}=\frac{\beta^{I I} c_{1} L}{K^{* I}}, \quad L_{51}=1 a \lambda_{3}^{I I}\left(b_{52}-b_{56}\right), \\
& L_{52}=-b_{55}\left(a^{2}+\lambda_{3}^{I I 2}\right), \quad \lambda_{3}=\frac{b_{51} a^{2}+\omega^{2}}{b_{51}}, \quad a_{5 n}=\frac{b_{52}}{\lambda_{n}^{2}-a^{2}-\omega^{2}}, \\
& g_{n}=\left(b_{53} \lambda_{n}^{2}-b_{54} a^{2}-1\right), \quad q_{n}=2 b_{5} 1 a \lambda_{n} \xi_{n}(n=1,2) .
\end{aligned}
$$




\section{Boundary conditions}

In this section, we determine the parameters $N_{j} ;(j=1,2,3,4,5)$ and $M_{j} ;(j=1,2,3)$. We should suppress the positive exponentials that are unbounded at infinity. Constants $N_{j} ;(j=1,2,3,4,5)$ and $M_{j}$; $(j=1,2,3)$ have to be selected such that boundary conditions at the surface $z=0$ take the form
(i) $t_{33}^{I}=t_{33}^{I I}-F e^{(\omega t+1 a x)}$,
(ii) $t_{31}^{1}-t_{31}^{I I}=0$,
(iii) $q_{33}^{I}=0$,
(iv) $q_{31}^{I}=0$,
(v) $u_{1}^{I}=u_{1}^{I I}$,
(vi) $u_{3}^{I}=u_{3}^{I I}$,
(vii) $T^{I}=T^{I I}$,
(viii) $K^{* I} \frac{\partial T^{I}}{\partial z}=K^{* I I} \frac{\partial T^{I I}}{\partial z}$

where $F$ is the magnitude of the mechanical force. Using Eqs (3.1)-(3.3) and (3.17), the boundary conditions given by Eq.(5.1) reduce to non-dimensional boundary conditions. With the help of Eqs (4.19)-(4.20) and (4.26)-(4.30), we obtain a system of eight non-homogeneous equations with eight unknowns $N_{m}$; $(m=1,2,3,4,5)$ and $R_{j} ;(j=1,2,3)$ as

$$
\begin{aligned}
& r_{51} N_{1}+r_{52} N_{2}+r_{53} N_{3}+r_{54} N_{4}-g_{51} R_{1}-g_{52} R_{2}-L_{51} R_{3}=N^{*}, \\
& s_{51} N_{1}+s_{52} N_{2}+s_{53} N_{3}-s_{54} N_{4}-q_{51} R_{1}-q_{52} R_{2}-L_{52} R_{3}=0, \\
& \gamma_{51} N_{1}+\gamma_{52} N_{2}+\gamma_{53} N_{3}+\gamma_{55} N_{5}=F_{52}, \\
& \eta_{51} N_{1}+\eta_{52} N_{2}+\eta_{53} N_{3}+\eta_{55} N_{5}=0, \\
& \imath a \alpha_{51} N_{1}+\imath a \alpha_{52} N_{2}+\imath a \alpha_{53} N_{3}+h_{4} N_{4}-\imath a a_{51} R_{1}-\imath a a_{52} R_{2}+\lambda_{3} R_{3}=-1 a l_{52}, \\
& h_{1} \alpha_{51} N_{1}+h_{2} \alpha_{52} N_{2}+h_{3} \alpha_{53} N_{3}-\imath a N_{4}+\lambda_{1} a_{51} R_{1}+\lambda_{2} a_{52} R_{2}+\imath a R_{3}=0, \\
& N_{1}+N_{2}+N_{3}-R_{1}-R_{2}=-l_{51}, \\
& h_{1} K^{* I} N_{1}+h_{2} K^{* I} N_{2}+h_{3} K^{* I} N_{3}-\lambda_{1} K^{* I I} R_{1}-\lambda_{2} K^{* I I} R_{2}=0 .
\end{aligned}
$$

The above eight Eqs (5.2)-(5.9) are solved by applying the inverse of matrix method and the values of eight constants $N_{m}(m=1,2,3,4,5)$ and $R_{j}(j=1,2,3)$ are obtained as 


$$
N_{j}=\left(\frac{\Delta_{5 m}}{\Delta}\right), \quad R_{j}=\left(\frac{\Delta_{6 j}}{\Delta}\right)
$$

where

$$
\begin{aligned}
& \Delta=f_{51}\left(\gamma_{52}-\gamma_{51}\right)\left(r_{53} h_{4}-1 a \alpha_{53} r_{54}\right), \quad \Delta_{51}=f_{51} f_{52}, \quad \Delta_{52}=f_{53} f_{52}, \\
& \Delta_{53}=f_{54} f_{52}, \quad \Delta_{54}=\left(f_{55}+f_{56}\right) f_{52}, \quad \Delta_{55}=g_{53}\left(g_{51}-g_{52}\right), \\
& \Delta_{61}=g_{54}\left(g_{51}-g_{52}\right), \quad \Delta_{62}=g_{55}\left(g_{51}-g_{52}\right), \quad \Delta_{63}=g_{56}\left(g_{51}-g_{52}\right), \\
& f_{51}=\left(1 a r_{54} a_{53}-r_{53} h_{4}\right)\left(F_{52}+\gamma_{52} l_{51}\right), \quad f_{52}=\left(\imath a q_{51} \lambda_{2} \eta_{55} K^{* I I}\right), \\
& f_{53}=\left(1 a r_{54} \alpha_{53}-r_{53} h_{4}\right)\left(F_{52}+\gamma_{51} l_{51}\right), \quad f_{54}=\left(\gamma_{52}-\gamma_{51}\right)\left(h_{4} N^{*}+r_{54} l_{52}\right), \\
& f_{55}=1 a\left(\alpha_{51} r_{52}-r_{51} \alpha_{52}\right)\left(l_{51} r_{53}+F_{52}\right), \quad f_{56}=1 a\left(r_{53} l_{52}+\alpha_{53} N^{*}\right)\left(\gamma_{51}-\gamma_{52}\right), \\
& g_{51}=1 a h_{3} K^{* I}\left(s_{51} \eta_{52}-s_{52} \eta_{51}\right), \quad g_{52}=s_{54} \eta_{53} h_{1} h_{2} K^{* I}\left(\alpha_{51}-\alpha_{52}\right), \\
& g_{53}=F_{52}\left(g_{52} \lambda_{3}+1 a a_{52} L_{51}\right), \quad g_{54}=l_{51} \gamma_{55}\left(g_{52} \lambda_{3}+1 a a_{52} L_{51}\right), \\
& g_{55}=\gamma_{55}\left(1 a l_{52} L_{51}-N^{*} \lambda_{3}\right), \quad g_{56}=-\gamma_{55} \mathrm{\imath} a\left(g_{52} l_{52}+a_{52} N^{*}\right), \quad N^{*}=F+F_{51} .
\end{aligned}
$$

\section{Particular cases}

\subsection{Generalized Thermoelastic Medium with Microtemperatures (GTM)}

Neglecting the effect of the internal heat source i.e., $Q \rightarrow 0$ in Eqs (4.19)-(4.20) we obtain the component of displacements, force stresses and temperature distribution in the generalized thermoelastic medium with microtemperatures (GTM).

\subsection{Generalized Thermoelastic Medium (GT)}

If we neglect both microtemperatures and the internal heat source, i.e., $\left(q_{i j}, Q=0\right)$ in Eqs (4.19)(4.25) we obtain the results for the generalized thermoelastic medium (GT).

\section{Numerical results}

In order to illustrate our theoretical results obtained in the proceeding section, we now consider a numerical example for which computational results are given. The results depict the variations of displacement components, force stresses and temperature distribution. For this purpose, we take the following values of physical constants (Steeb et al. [21])

$$
\lambda^{\diamond}=2.17 \times 10^{10} \mathrm{Nm}^{-2}, \quad \mu^{\diamond}=3.2784 .0 \times 10^{10} \mathrm{Nm}^{-2}, \quad \rho^{\diamond}=1.74 \times 10^{3} \mathrm{kgm}^{-3},
$$




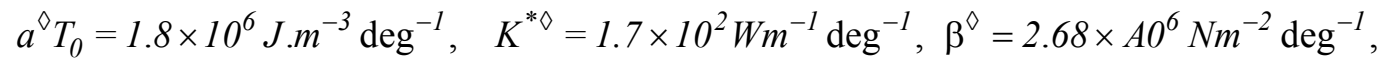

$$
\begin{aligned}
& k_{1}=2 \times 10^{10} \mathrm{Wm}^{-1}, \quad k_{2}=0.1 \times 10^{10} \mathrm{Wm}^{-1}, \quad k_{3}=04 \times 10^{10} \mathrm{Wm}^{-1}, \quad k_{4}=0.3 \times 10^{10} \mathrm{Wm}^{-1}, \\
& k_{5}=0.5 \times 10^{10} \mathrm{Wm}^{-1}, \quad k_{6}=0.7 \times 10^{10} \mathrm{Wm}^{-1} .
\end{aligned}
$$

The computations are carried out for the value of non-dimensional time $t=0.2$ in the range $0 \leq x \leq 10$ and on the surface $z=1.2$. The numerical values for displacements $\left(u_{1}{ }^{I}, u_{3}{ }^{I}\right)$, force stresses $\left(t_{31}{ }^{I}, t_{33}{ }^{I}\right)$ and temperature distribution $T^{\diamond}$ are shown in Figs 1-5 for the mechanical force with magnitude $F=1.0, \omega=\omega_{0}+1 \varsigma, \omega_{0}=2.0, \varsigma=0.1, a=0.9$ and $Q=2$ for a

(a) generalized thermoelastic medium with microtemperature having an internal heat source (GTMI) by a solid line (i.e., $q_{i j}, Q_{1} \neq 0$ ).

(b) generalized thermoelastic medium with microtemperature (GTM) by a solid line with centered symbols (*) (i.e., $q_{i j} \neq 0, Q_{1}=0$ ).

(c) generalized thermoelastic medium (GT) by a dashed line with centered symbols $(*)$ (i.e., $q_{i j}$, $\left.Q_{1}=0\right)$.

\section{Discussions}

The variation of the tangential displacement $u_{l}^{I}$ for GT is highly oscillating in nature in comparison to the variations obtained for GTMI and GTM. These variations of the tangential displacement $u_{1}^{I}$ are shown in Fig.1. It is quite evident from Fig.2 that the variations of the normal displacement $u_{3}{ }^{I}$ is least oscillatory for GT. The variations of the normal displacement $u_{3}{ }^{I}$ for GTMI and GTM are opposite in nature with fluctuating values. The variations of the tangential force stress $t_{31}{ }^{I}$ with the distance $x$ is depicted in Fig.3. Initially, the value of the tangential force stress $t_{31}{ }^{I}$ for GT starts with a sharp increase in the range $0 \leq x \leq 2.0$ and then follows an oscillatory pattern with reference to $x$. Further the tangential force stress $t_{31}^{I}$ shows small variations close to zero in the whole range for GTM. Figure 4 depicts the variations of the normal force stress $t_{33}{ }^{I}$ with the distance $x$. The variations of the normal force stress $t_{33}{ }^{I}$ are very closely related with each other having different magnitude for GTMI and GT. Also, the value of the normal force stress $t_{33}{ }^{I}$ for GTM lie in a very short range. Further discussions are not required for the variations obtained in the case of the temperature distribution $T^{I}$, since it is evident from Fig.5 that these variations are similar to the variations obtained in the case of the normal displacement $u_{3}{ }^{I}$ with difference in their magnitude. 


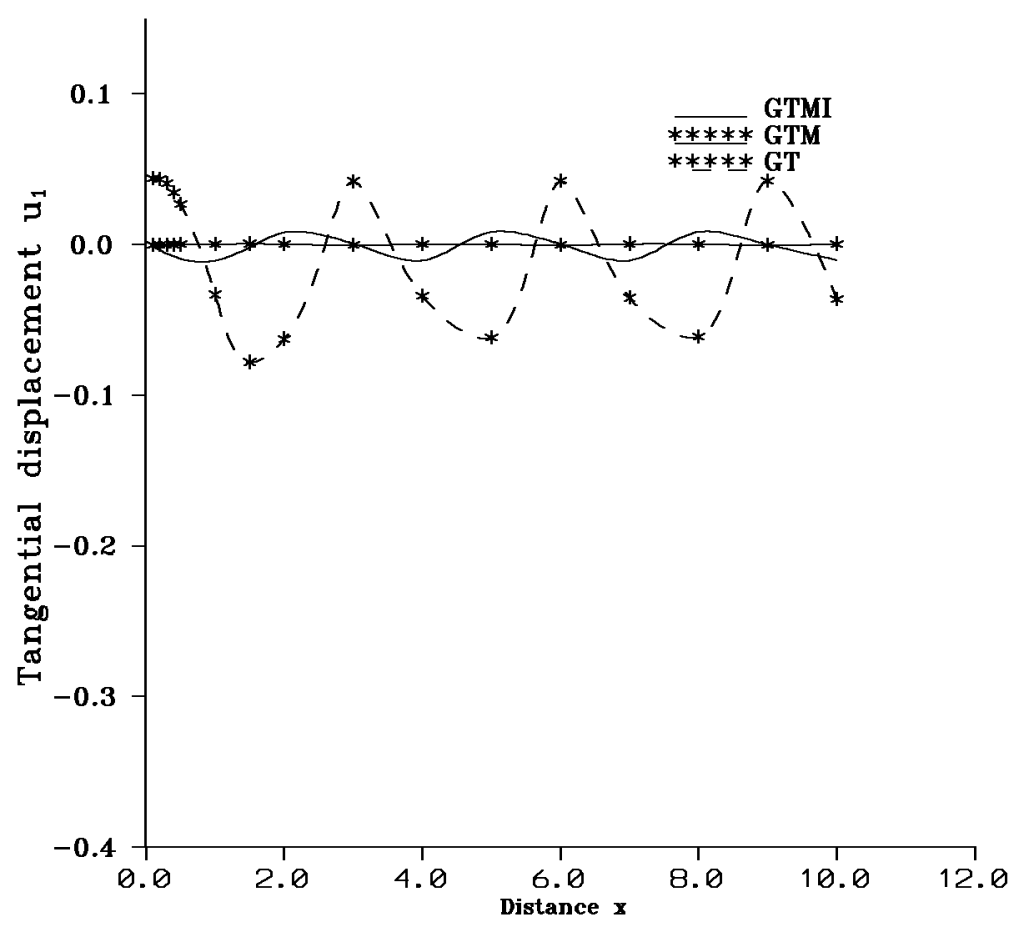

Fig.1. Variations of tangential displacement $u_{1}$ with distance $x$.

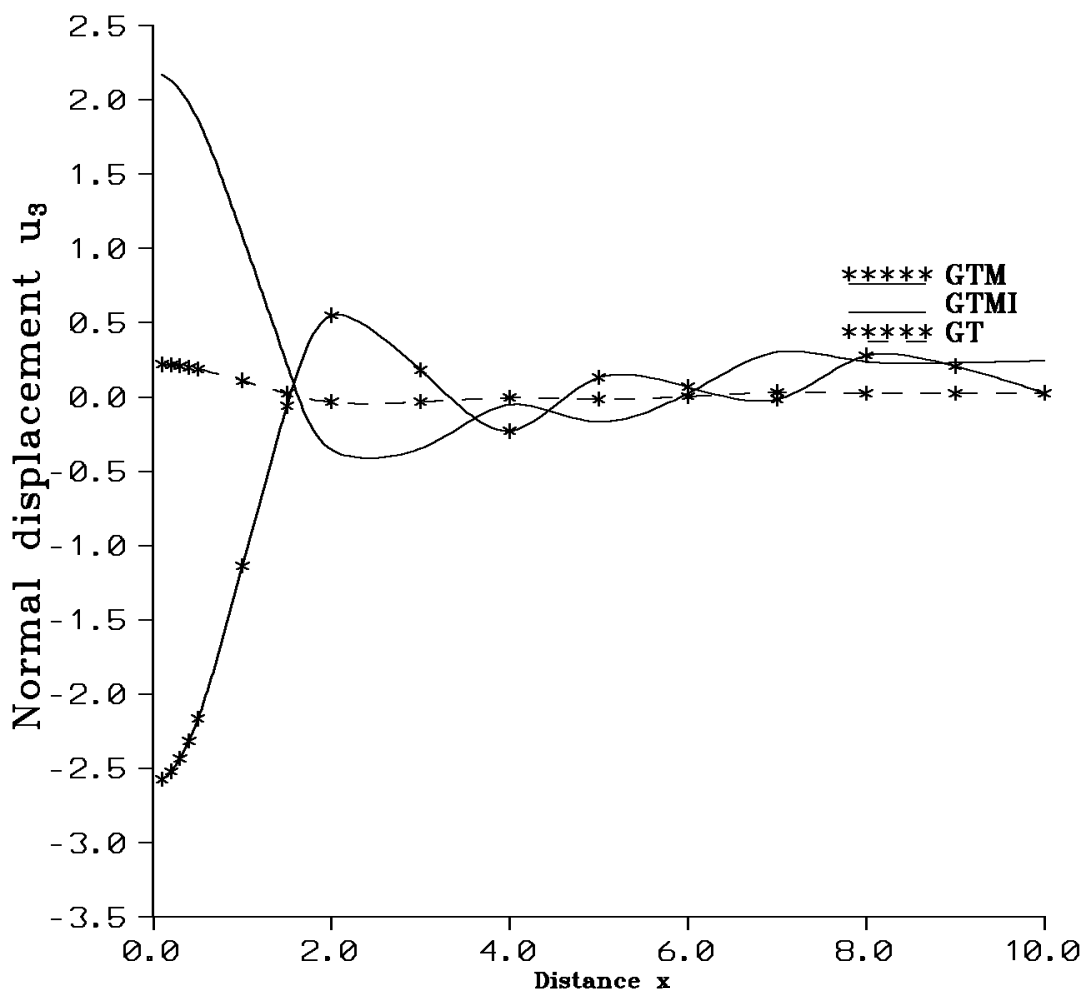

Fig.2. Variations of normal displacement $u_{31}$ with distance $x$. 


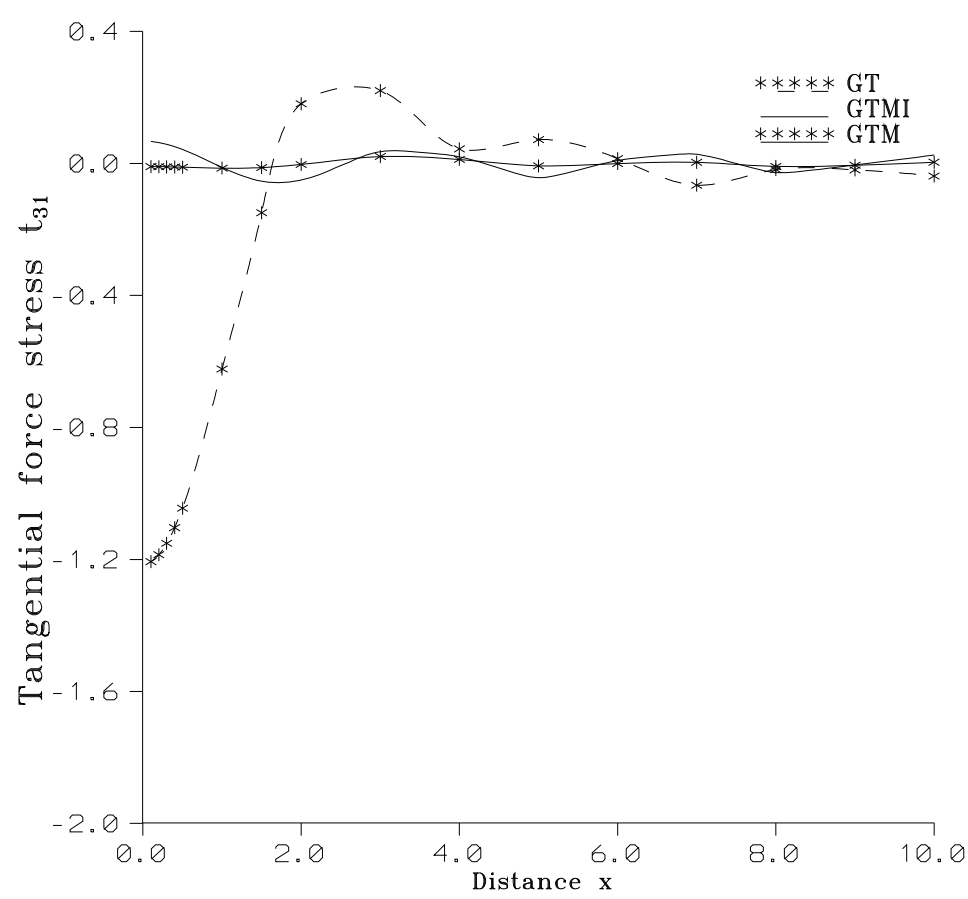

Fig.3. Variations of tangential force stress $t_{31}$ with distance $x$.

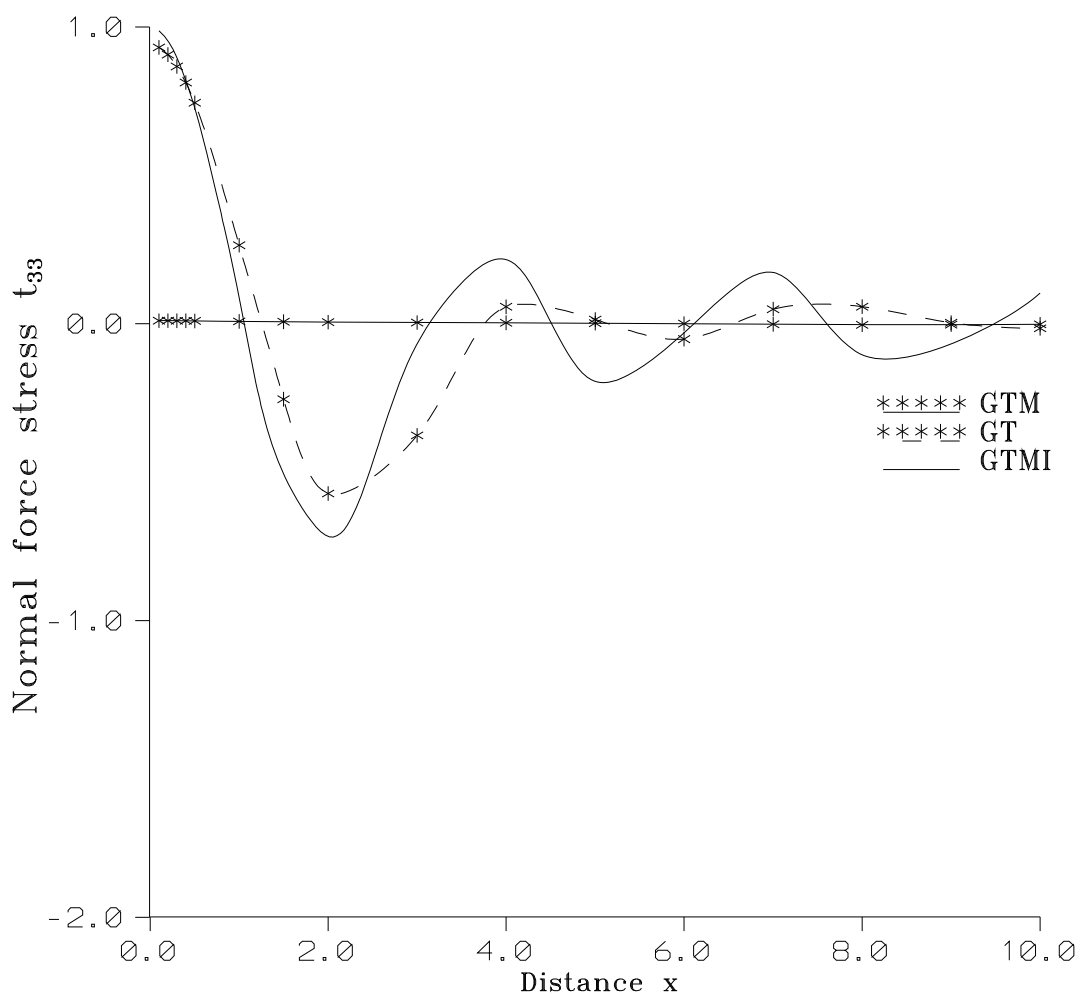

Fig.4. Variations of normal force stress $t_{33}$ with distance $x$. 


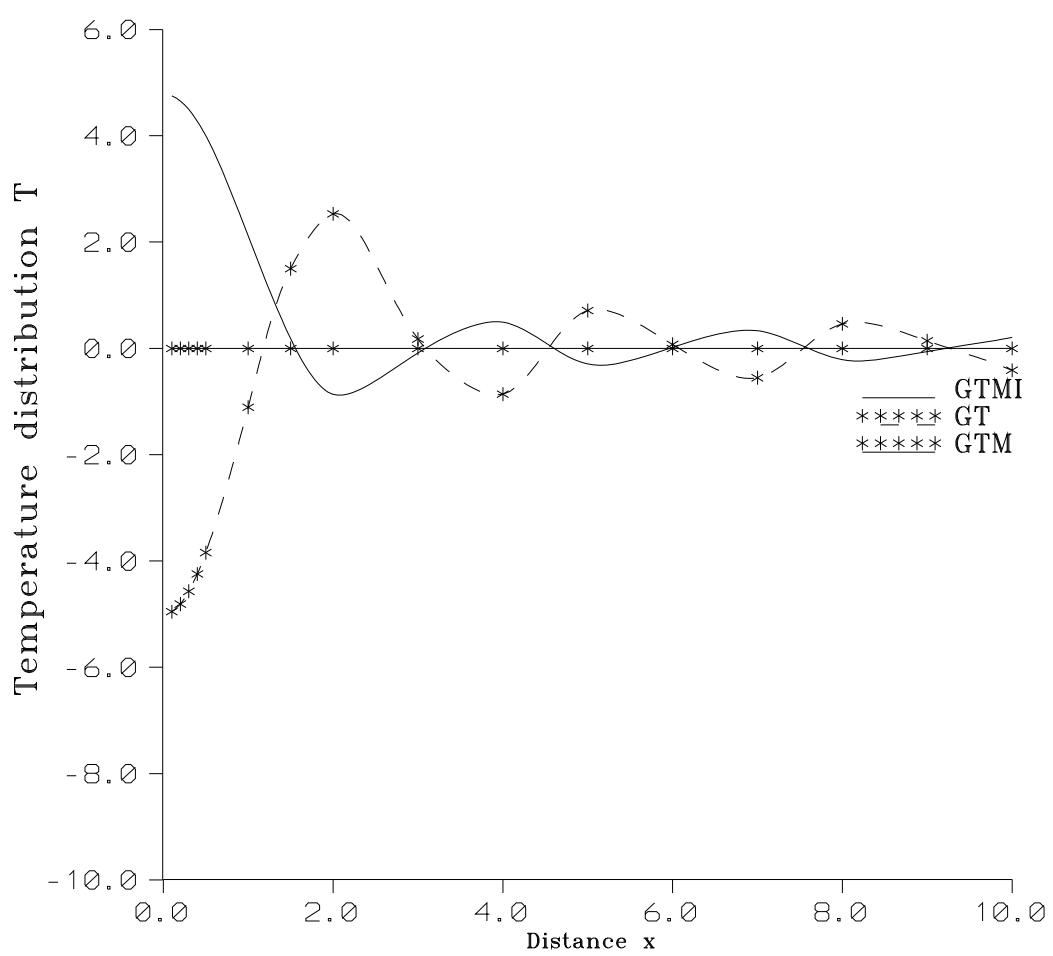

Fig.5. Variations of temperature distribution $T$ with distance $x$.

\section{Conclusion}

Both microtmperatures and internal heat source have a significant effect on displacements, force stresses and temperature distribution. The variations of the normal displacement $u_{3}$ and temperature distribution $T$ are similar in nature with difference in magnitude. The variations of all the quantities are least oscillating for GTM. The normal mode analysis used in this article is applicable to a wide range of problems in different branches. This method gives exact solutions without any assumed restrictions on either the temperature or stress distributions. It helps us to study the effect of a heat source in the medium and the deformation caused in the medium due to the heat source.

\section{Nomenclature}

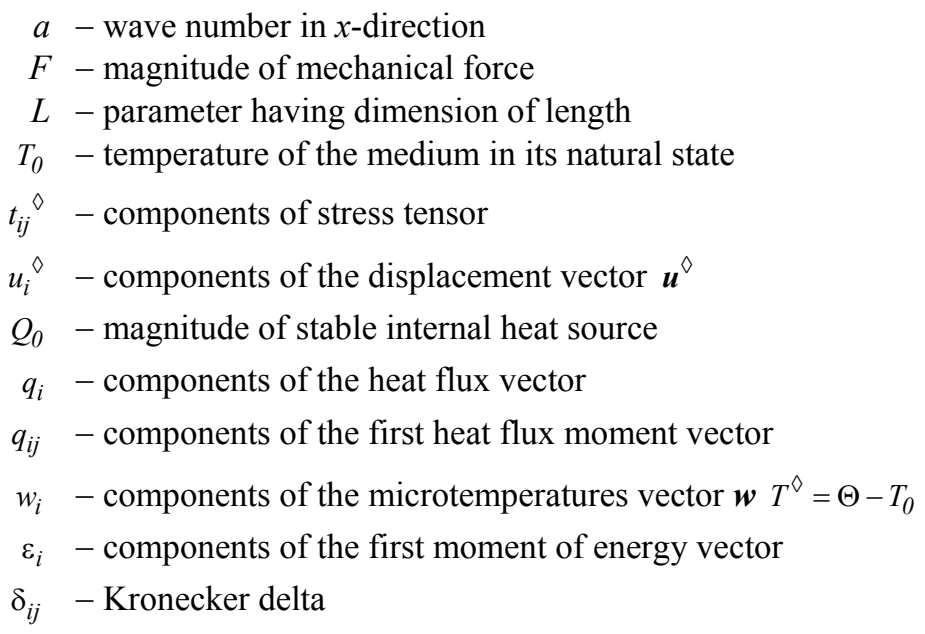




$$
\begin{aligned}
\eta^{*} & - \text { entropy per unit mass } \\
\Theta & - \text { temperature at time } t \\
\lambda^{\diamond}, \mu^{\diamond}, \beta^{\diamond}, a^{\diamond}, b, K^{* \diamond}, & - \text { constant constitutive coefficents } \\
k_{i}(j=1,2, \ldots \sigma) & \\
\rho^{\diamond} & - \text { reference mass density of the medium } \\
{\left[\bar{\phi}^{I}, \bar{\psi}^{I}, \varphi_{1}, \psi_{1}, \bar{T}^{I}, \bar{t}_{i j}^{I}, \bar{q}_{i j}\right] } & - \text { magnitude of the functions } \\
\omega & - \text { complex time constant }
\end{aligned}
$$

\section{References}

[1] Grot R. (1969): Thermodynamics of a continuum with microstructure. - International Journal of Engineering Science, vol.7, pp.801-814.

[2] Iesan D. and Quintanilla R. (2000): On a theory of thermoelasticity with microtemperatures. - Journal of Thermal Stresses, vol.23, pp.199-215.

[3] Riha P. (1976): On the microcontinuum model of heat conduction in materials with inner structure. - International Journal of Engineering Science, vol.14, pp.529-535.

[4] Riha P. (1975): On the theory of heat conducting micropolar fluids with microtemperatures. - Acta Mechanics, vol.23, pp.1-8.

[5] Riha P. (1977): Poiseuille flow of microthermopolar fluids. - Acta Technology, vol.22, pp.602-614.

[6] Iesan D. (2001): On a theory of micromorphic elastic solids with microtemperatures. - Journal of Thermal Stresses, vol.24, pp.737-752.

[7] Iesan D. (2002). On the theory of heat conduction in micromorphic continua. - International Journal of Engineering Science, vol.40, pp.1859-1878.

[8] Iesan D. (2007): Thermoleasticity of bodies with microstructure and microtemperatures. - International Journal of Solids and Structures, vol.44, pp.8648-8662.

[9] Iesan D. and Quintanilla R. (2009): On thermoelastic bodies with inner structure and microtemperatures. - Journal of Mathematical Analysis and Applications, vol.354, No.1, pp.12-23.

[10] Svanadze M. (2003): Boundary value problems of the theory of thermoelasticity with microtemperatures. Proceeding in Applied Mathematics and Mechanics, vol.3, pp.188-189.

[11] Svanadze M. (2004): Fundamental solutions of the equations of the theory of thermoelasticity with microtemperatures. - Journal of Thermal Stresses, vol.27, pp.151-170.

[12] Scalia A. and Svanadze M. (2006): On the representations of solutions of the theory of thermoelasticity. - Journal of Thermal Stresses, vol.29, pp.849-863.

[13] Scalia A. and Svanadze M. (2009): Potential method in the linear theory of thermoelasticity with microtemperatures. - Journal of Thermal Stresses, vol.32, pp.1024-1042.

[14] Scalia A., Svanadze M. and Tracina R. (2010): Basic theorems in the equilibrium theory of thermoelasticity with microtemperatures. - Journal of Thermal Stresses, vol.33, pp.721-753.

[15] Iesan D. (2009): Singular surfaces in the theory of thermoelasticity with microtemperatures. - Journal of Thermal Stresses, vol.32, pp.617-621.

[16] Iesan D. and Scalia A. (2010): Plane deformation of elastic bodies with microtemperatures. - Mechanics Research Communications, vol.37, No.7, pp.617-621.

[17] Yang L. and Huang L. (2010): Propagation of singularities for Cauchy problems of semilinear thermoelastic systems with microtemperatures. - Mathematische Nachrichten, vol.283, pp.615-629. 
[18] Aouadi M. (2008): Some theorems in the isotropic theory of microstretch thermoelasticity with microtemperatures. - Journal of Thermal Stresses, vol.31, pp.649-662.

[19] Svanadze M. and Tracina R. (2011): Representations of solutions in the theory of thermoelasticity with microtemperatures for microstretch solids. - Journal of Thermal Stresses, vol.34, pp.161-178.

[20] Chirita S., Ciarletta M. and Apice C.D. (2013): On the theory of thermoelasticity with microtemperatures. Journal of Mathematical Analysis and Applications, vol.397, pp.349-361.

[21] Steeb H., Singh J. and Tomar S.K. (2013): Time harmonic waves in thermoelastic material with microtemperatures. - Mechanics Research Communication, vol.48, pp.8-18.

[22] Kumar R. and Kaur M. (2014): Reflection and refraction of plane waves at the interface of an elastic solid and microstretch thermoelastic solid with microtemperatures. - Archive of Applied Mechanics, vol.84, No.4, pp.571590.

[23] Ailawalia P., Sachdeva S.K. and Pathania D. (2015): Two dimensional deformation in microstretch thermoelastic half space with microtemperatures and internal heat source. - Cogent Mathematics, vol.2, pp.1-15.

[24] Othman M.I.A, Tantawi R.S. and Hilal M.I.M. (2016): Effect of initial stress and gravity field on micropolar thermoelastic solid with microtemperatures. - Journal of Theoretical and Applied Mechanics, vol.54, No.3, pp.847-857.

Received: January 2, 2017

Revised: October 11, 2017 